\title{
Medroxyprogesterone acetate and levonorgestrel increase genital mucosal permeability and enhance susceptibility to genital herpes simplex virus type 2 infection
}

\author{
NE Quispe Calla ${ }^{1}$, RD Vicetti Miguel ${ }^{1}$, PN Boyaka ${ }^{2}$, L Hall-Stoodley ${ }^{1}$, B Kaur ${ }^{3}$, W Trout ${ }^{4}$, SD Pavelko ${ }^{1}$ and \\ TL Cherpes ${ }^{1,4}$
}

Depot-medroxyprogesterone acetate (DMPA) is a hormonal contraceptive especially popular in areas with high prevalence of HIV and other sexually transmitted infections (STI). Although observational studies identify DMPA as an important STI risk factor, mechanisms underlying this connection are undefined. Levonorgestrel (LNG) is another progestin used for hormonal contraception, but its effect on STI susceptibility is much less explored. Using a mouse model of genital herpes simplex virus type 2 (HSV-2) infection, we herein found that DMPA and LNG similarly reduced genital expression of the desmosomal cadherin desmoglein-1 $\alpha$ (DSG1 $\alpha$ ), enhanced access of inflammatory cells to genital tissue by increasing mucosal epithelial permeability, and increased susceptibility to viral infection. Additional studies with uninfected mice revealed that DMPA-mediated increases in mucosal permeability promoted tissue inflammation by facilitating endogenous vaginal microbiota invasion. Conversely, concomitant treatment of mice with DMPA and intravaginal estrogen restored mucosal barrier function and prevented HSV-2 infection. Evaluating ectocervical biopsy tissue from women before and 1 month after initiating DMPA remarkably revealed that inflammation and barrier protection were altered by treatment identically to changes seen in progestin-treated mice. Together, our work reveals DMPA and LNG diminish the genital mucosal barrier; a first-line defense against all STI, but may offer foundation for new contraceptive strategies less compromising of barrier protection.

\section{INTRODUCTION}

The long-term preponderance of women living with HIV in sub-Saharan Africa provided strong impetus to determine the factors promoting male-to-female sexual transmission of the virus. ${ }^{1}$ Although numerous observational studies identified the injectable hormonal contraceptive depot-medroxyprogesterone acetate (DMPA) as a significant risk factor for acquisition of HIV and other sexually transmitted infections (STI), other studies failed to detect this association. ${ }^{2-5}$ However, close connections between hormonal contraceptive use and behavior (i.e., the higher frequency of unprotected sex among individuals using hormonal contraception) may have confounded data interpretation from these studies and thus contributed to the inconsistency of their conclusions. ${ }^{6-8}$ Levonorgestrel (LNG) is a progestin used in intrauterine devices (IUD) that provide long-acting reversible contraceptives (LARC), but its effect on STI susceptibility is much less explored than DMPA, both experimentally and clinically.

Because of the limitations of observational studies, there is interest in conducting randomized controlled trials to more precisely define the relationships between STI susceptibility and use of DMPA, LNG, and other LARC. ${ }^{9}$ However, accrued evidence already substantiating the connection between DMPA use and STI acquisition raises concerns for random assignment

\footnotetext{
${ }^{1}$ Department of Microbial Infection and Immunity, Columbus, Ohio, USA. ²Department of Veterinary Biosciences, The Ohio State University College of Veterinary Medicine, Columbus, Ohio, USA. '3 Department of Neurological Surgery, James Comprehensive Cancer Center, The Ohio State University Medical Center, Columbus, Ohio, USA and ${ }^{4}$ Department of Obstetrics and Gynecology, The Ohio State University College of Medicine, Columbus, Ohio, USA. Correspondence: NE Quispe Calla Calla or RD Vicetti Miguel or TL Cherpes (quispecalla.1@osu.edu or vicettimiguel.1@osu.edu or cherpes.1@osu.edu )
} 
of women to specific LARC therapies, ${ }^{10}$ while prior studies randomizing women to receive DMPA reported suboptimal rates of participation and retention. ${ }^{11,12}$ Moreover, it will be challenging for any type of clinical investigation, including randomized controlled trials, to identify the biological mechanisms by which LARC increase susceptibility for STI acquisition. $^{13,14}$ Although genital mucosal epithelium thinning, enhanced tissue inflammation, suppressed cell-mediated immune responses, and altered vaginal microbiota were proposed to connect DMPA with enhanced STI acquisition, these putative mechanisms remain experimentally unproven. $^{15,16}$ The objective of this investigation was therefore to perform a series of experiments using murine models and clinical genital tract specimens that identified specific host antiviral defense mechanisms that are compromised by two progestins currently utilized in LARC.

\section{RESULTS}

DMPA and LNG increased mouse susceptibility to herpes simplex virus type 2 infection

Antecedent DMPA treatment enhances infectivity of viruses and bacteria in various rodent and non-human primate models of female genital tract infection. ${ }^{17-20}$ To define mechanisms responsible for these experimental observations, in the current investigation we utilized a system in which DMPA makes mice highly susceptible to intravaginal (ivag) herpes simplex virus (HSV) infection. The herpes simplex viruses (HSV-1 and HSV-2) are human pathogens that infect mucocutaneous surfaces. Initial replication of these viruses in epithelial cells causes a productive lytic infection, which quickly disseminates to the central nervous system. In the murine model of genital HSV-2 infection, lytic infection causes extensive genital pathology that is soon followed by the development of fatal encephalopathic changes.

Because antecedent DMPA administration makes mice uniformly susceptible to genital HSV acquisition, ${ }^{21,22}$ we used a fluorescently tagged HSV-1 (ref. 23) to ivag infect C57BL/6J mice that were in estrus (i.e., the estrous cycle stage with lowest endogenous progesterone levels) or 5 days after DMPA treatment. Using confocal microscopy, we saw that HSV-1 had spread precipitously throughout the vaginal mucosa of DMPA-treated mice by $24 \mathrm{~h}$ after infection, whereas virus was undetectable at this same time point after infection in mice infected during estrus (Figure 1a). We then assessed the development of external genital pathology and fatal encephalopathy after ivag HSV-2 infection of mice in estrus, diestrus (i.e., the estrous cycle stage with highest levels of endogenous progesterone), or 5 days after DMPA administration. And as predicted by enhanced dissemination of fluorescent HSV-1 in DMPA-treated mice, infection was uniformly lethal after DMPA treatment, while morbidity and mortality were absent or more incrementally increased among mice infected during estrus or diestrus, respectively (Figure 1b,c).

Although MPA binds the progesterone receptor (PR) and the glucocorticoid receptor (GR), LNG (the progestin commonly used in IUD providing $\mathrm{LARC}^{24}$ ) more specifically binds the PR. ${ }^{25,26}$ Utilizing the same mouse model of HSV-2 infection (but treating with LNG rather than DMPA), we thus could newly explore the role of a more specific PR agonist in altering susceptibility to a genital viral pathogen. In this study, we found that LNG enhanced dissemination of fluorescently-tagged HSV-1 and susceptibility to ivag HSV-2 infection in ways identical to results seen with DMPA (Figure 1a,c). We then used a similar strategy in this mouse model to evaluate the effects of administering methylprednisolone (MePRDL) (a pure GR agonist), progesterone (Pg) (a PR agonist with negligible GR binding), or DMPA and mifepristone (RU486) (a GR and PR antagonist) before infection. Analogous to the effects of DMPA or LNG, infection was uniformly lethal in Pg-treated mice (Figure 1b,c). Conversely, no morbidity or mortality developed in mice treated with DMPA and RU486 or MePRDL (Figure $\mathbf{1 b}, \mathbf{c}$ ). Interestingly, separate analyses of vaginal tissues from mice in estrus, and mice treated with DMPA, LNG, or MePRDL, showed that MePRDL alone modulated expression of multiple glucocorticoid-regulated genes $^{27}$ (specifically, increased expression of glucocorticoidinduced leucine zipper, glucocorticoid-regulated kinase 1, dual specificity protein phosphatase 1 , and decreased expression of the GR) (Supplementary Figure S1 online). Taken together, these initial studies newly revealed that enhanced susceptibility of mice to ivag HSV-2 infection is a class effect of progestins (including MPA and LNG), but not an effect of the GR agonist MePRDL.

\section{DMPA and LNG increased permeability of mouse genital mucosal epithelium}

Because external genital pathology developed within 3 days after DMPA- or LNG-treated mice were genitally infected (i.e., before formation of HSV-specific adaptive immunity), we next focused on the effects of these progestins on innate immunity in the female genital tract, specifically the mucosal barrier. In initial studies, we explored the possibility that the sizeable increases in mortality among progestin-treated mice compared with mice infected in diestrus (100 vs. 25\%, respectively) (Figure 1c) were associated with increased genital mucosal thinning. However, we found that vaginal mucosal thickness was comparable between mice in diestrus and after progestin treatment (Figure 2a and Supplementary Figure S2), indicating that mucosal thinning was not solely responsible for the conspicuously higher mortality in progestin-treated mice.

On the basis of these results, we next explored the progestinmediated effects on genital mucosal permeability by intravaginally administering $457 \mathrm{Da}$ and $70 \mathrm{KDa}$ fluorescent molecules to estrus-stage, diestrus-stage, and DMPA- or LNG-treated mice. Forty-five minutes later, mice were killed and the genital tracts excised to assess fluorescent molecule penetration into the vaginal mucosa by confocal microscopy. In results wholly consistent with mouse survival after HSV-2 infection (Figure 1c), these images showed that permeability to the $457-\mathrm{Da}$ fluorescent molecule was moderately increased in 
a
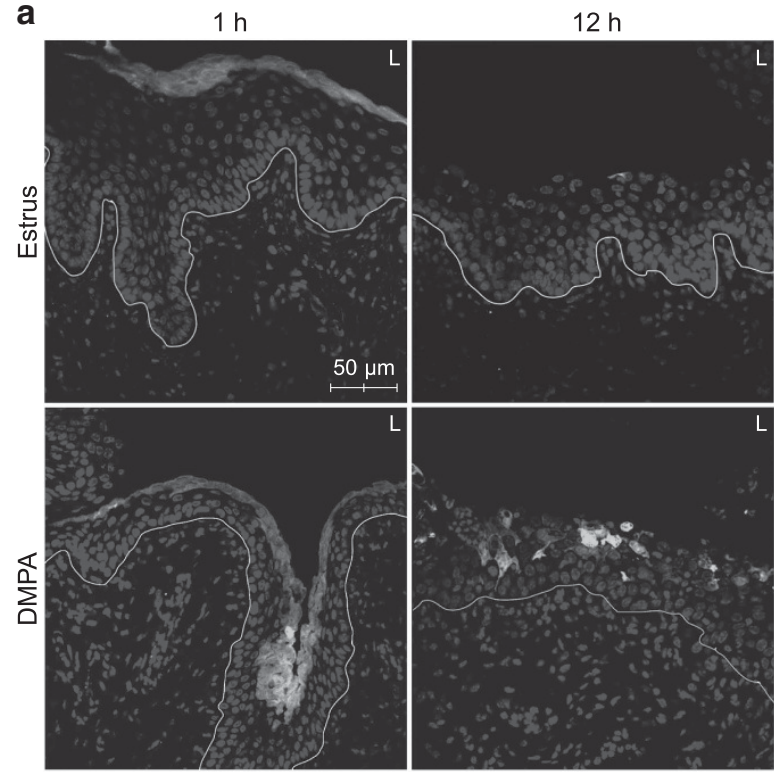

$12 \mathrm{~h}$

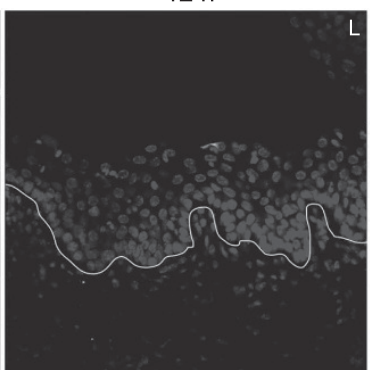

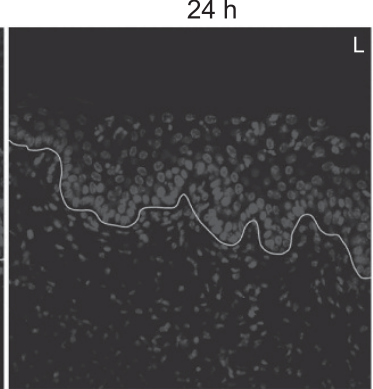

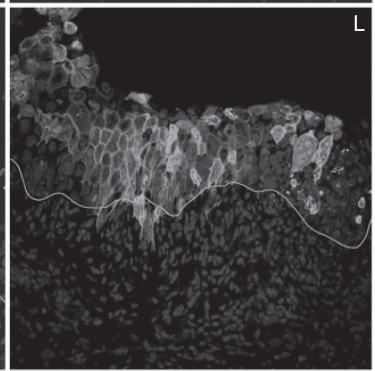

b

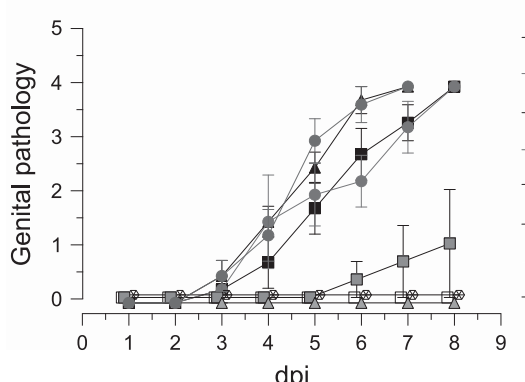

- $\mathrm{Pg}$

- LNG sc

- LNG ivag

- DMPA

$\triangle$ Estrus

$\square$ Diestrus

$\square$ MePRDL

DMPA +

RU486

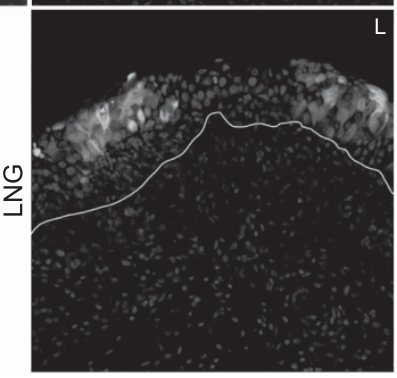

C

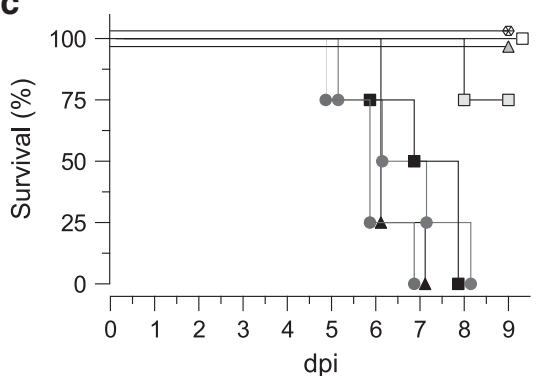

Figure 1 MPA and LNG increased mouse susceptibility to ivag HSV-2 infection. (a) Untreated mice in estrus and DMPA- or LNG-treated mice were ivag infected with $3 \times 10^{6} \mathrm{pfu}$ HSV-1q-GPF, and killed 1, 12, or $24 \mathrm{~h}$ later. Examination of excised vaginal tissue by confocal microscopy showed widespread viral dissemination in the mucosa of DMPA- and LNG-treated mice, while virus failed to extend beyond superficial mucosal surfaces of mice infected during estrus (L indicates vaginal lumen); HSV-1q-GPF (green); DAPI (blue). (b, c) In separate studies, untreated mice in estrus or diestrus; DMPA-treated, Pg-treated, DMPA and RU486-treated mice; mice treated with LNG (s.c. or ivag); or MePRDL-treated mice were infected with $10^{4}$ pfu HSV-2 and observed daily for genital pathology and mortality. Consistent with fluorescent HSV data (a), Kaplan-Meier survival curve showed that DMPA, LNG s.c., LNG ivag, and Pg treatments produced $100 \%$ mortality, while no mice infected in estrus, treated with DMPA and RU486, or treated with MePRDL developed morbidity or mortality (data representative of two independent experiments containing five mice per group). To compare scores for the development of genital pathology, areas under the curve were calculated for individual mice, and multiple group comparisons performed using Kruskal-Wallis test with Dunn's post hoc test (diestrus compared with estrus, $P=0.0243$; for all other groups compared with estrus, $P<0.0001$ ). For Kaplan-Meier survival curve data, log-rank tests were used to compare cumulative survival incidence after ivag HSV-2 infection (diestrus compared with estrus, $P=0.0165$; for all other groups compared with estrus, $P<0.0001$ ). DAPI, 4,6-diamidino-2-phenylindole; DMPA, depot-medroxyprogesterone acetate; HSV-2, herpes simplex virus type 2; ivag, intravaginally; LNG, levonorgestrel; MePRDL, methylprednisolone; s.c., subcutaneously. A full color version of this figure is available at the Mucosal Immunology journal online.

diestrus-stage mice but dramatically increased by DMPA or LNG treatment (Figure 2b). Conversely, permeability to these same molecules was absent in untreated mice in estrus and mice treated with MePRDL or both DMPA and RU486 (Figure 2b). Following-up on these results, we evaluated whether progestin treatment similarly facilitated tissue penetration of leukocytes, immune cells involved in the transmission of HIV and other STI. ${ }^{28}$ And congruent with enhanced penetration of the lowmolecular weight (LMW) molecules, we saw that fluorescentlylabeled syngeneic splenocytes readily infiltrated vaginal mucosa 

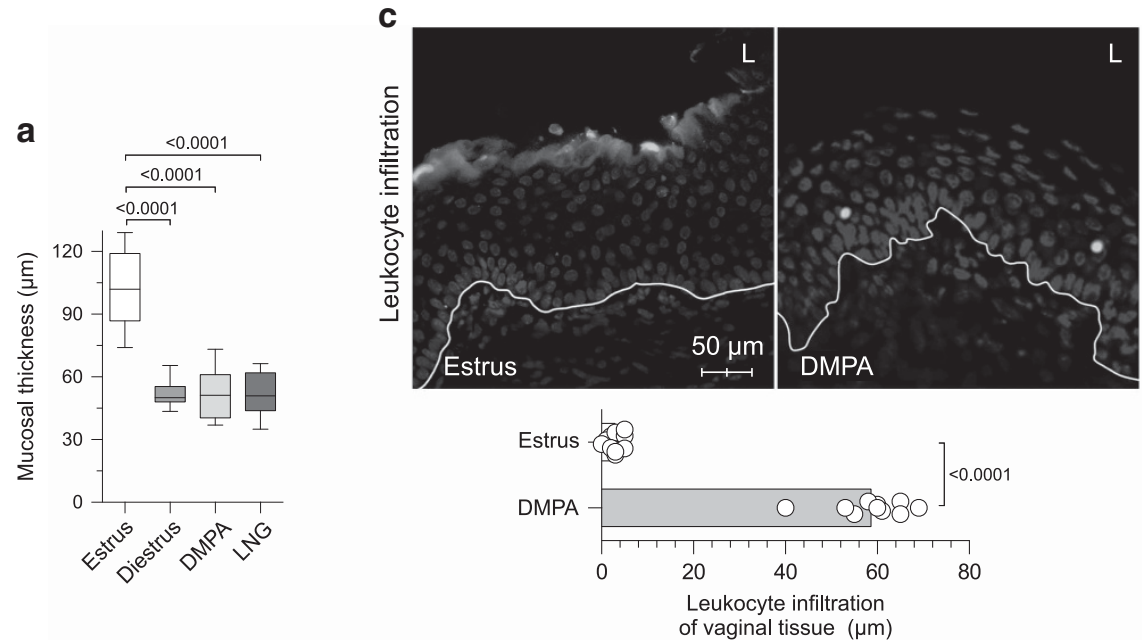

b

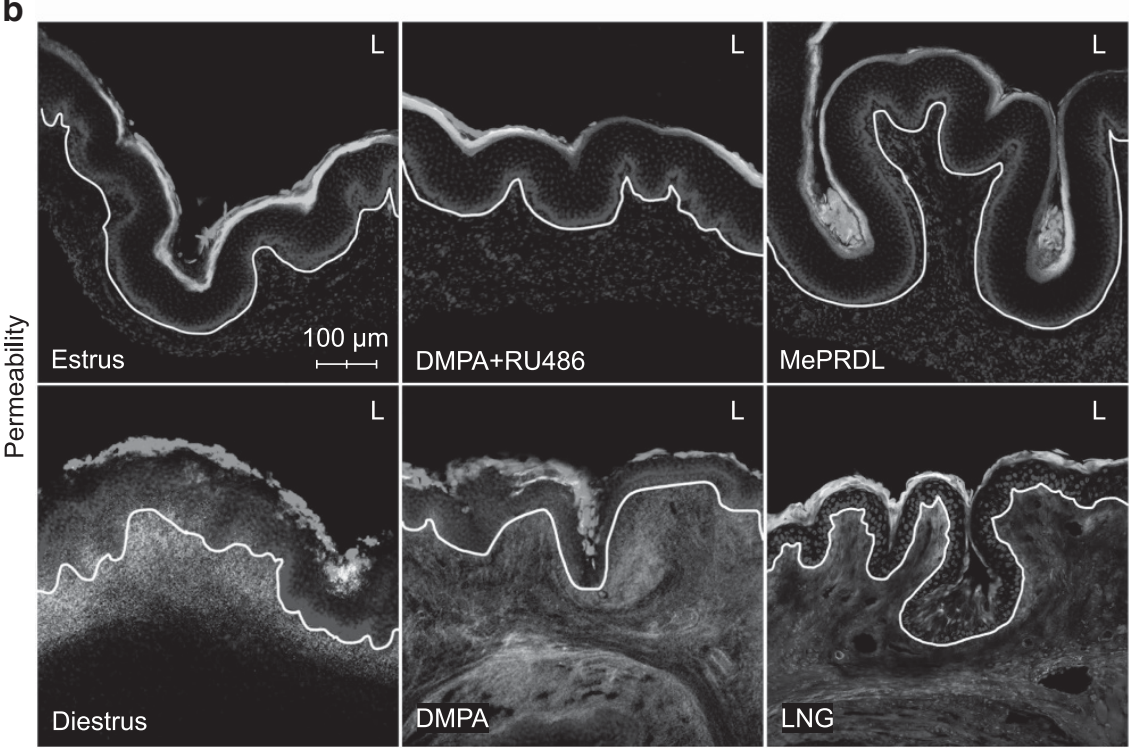

Figure 2 DMPA and LNG similarly increased genital mucosal permeability in mice. (a) Vaginal mucosal thicknesses were equivalent in mice in diestrus and DMPA- or LNG-treated mice, indicating that differential epithelial thinning had not caused the sizeable differences in mortality after ivag HSV-2 infection (Figure 1b,c) (H\&E-stained vaginal tissue sections from four mice were assessed per condition and five fields per section evaluated by light microscopy at $\times 200$ magnification). (b) Groups of mice treated as described in Figure 1b were anesthetized and ivag administered PBS containing Lucifer yellow (LY) (457.2 Da) (green) and 70 KDa dextran-Texas Red (DR) (red). Forty-five minutes later, mice were killed and vaginal tissue processed and counterstained with DAPI (blue) for confocal microscopic analysis. Although neither fluorescent molecule entered vaginal tissue of mice in estrus or mice treated with DMPA and RU486 or MePRDL, LY entered the vaginal mucosa of mice in diestrus and entry was more pervasive in the vaginal mucosa of DMPA- or LNG-treated mice (representative images from two independent experiments with five animals per condition). (c) Anesthetized mice in estrus and DMPA-treated mice were ivag administered $2 \times 10^{6}$ CFSE-labeled syngeneic splenocytes (green), and $12 \mathrm{~h}$ later vaginal tissue treated as detailed in Methods. Confocal microscopy analysis showed significantly deeper penetration of CFSE-labeled splenocytes into the vaginal mucosa of DMPA-treated mice vs. mice in estrus. Representative images and data quantification from two independent experiments with five animals per condition are shown (L denotes vaginal lumen). Between-group comparisons of vaginal mucosal thickness were made with the Kruskal-Wallis and Dunn's post hoc tests, and differences in splenocyte entry into vaginal mucosa were made with the unpaired Student's $t$-test. CFSE, carboxyfluorescein succinimidyl ester; DAPI, 4,6-diamidino-2-phenylindole; DMPA, depot-medroxyprogesterone acetate; HSV-2, herpes simplex virus type 2; LNG, levonorgestrel; MePRDL, methylprednisolone. A full color version of this figure is available at the Mucosal Immunology journal online.

tissue of DMPA-treated mice but not mice in estrus (Figure 2c). These latter results demonstrated that progestin-mediated increases in genital mucosal permeability were not restricted to facilitating diffusion of LMW molecules across the mucosal barrier, but extended to increasing genital epithelial surface permeability to considerably larger-sized cells that enter mucosal tissue by active processes.
To further explore progestin-mediated increases in genital mucosal permeability, we harvested vaginal tissue from mice in estrus, diestrus, and DMPA- or LNG-treated mice, and measured expression of the desmosomal cadherins desmoglein-1 $\alpha$ (DSG1 $\alpha$ ) and desmocollin (DSC1). These cell-cell adhesion molecules are present in female genital tract epithelium, ${ }^{29,30}$ and shown important for maintaining the 

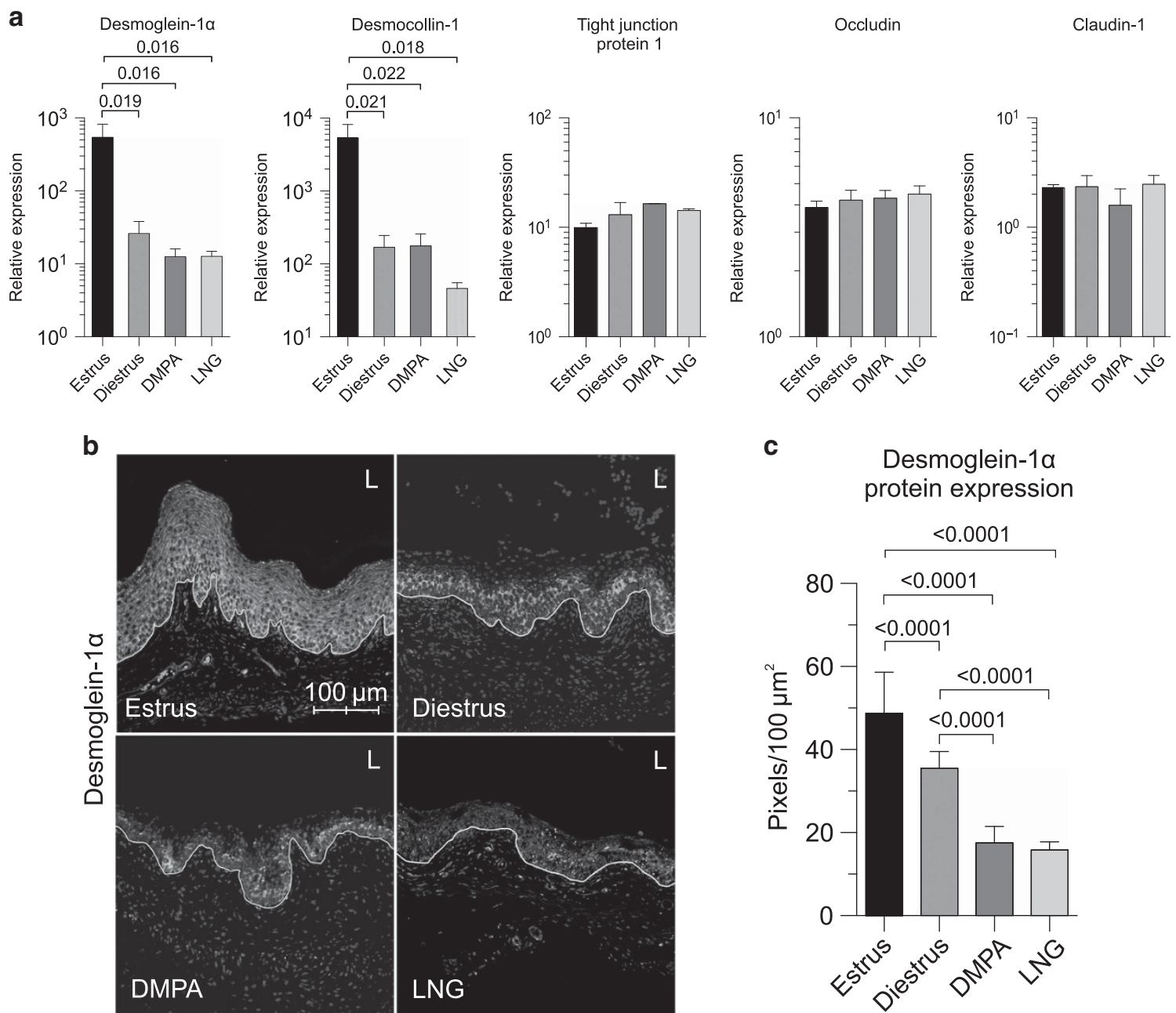

C

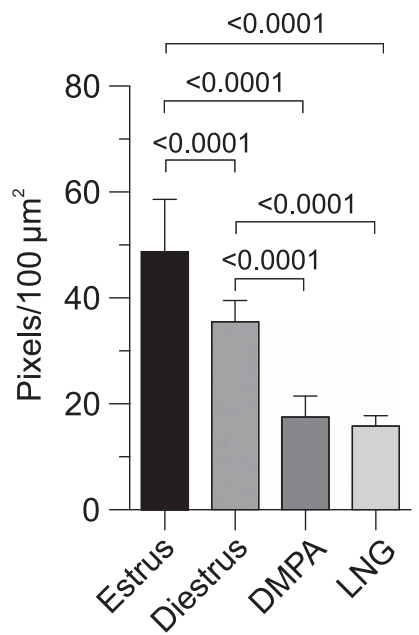

Figure 3 DMPA and LNG reduced vaginal tissue expression of the desmosomal cadherins DSG1 $\alpha$ and DSC1. (a) Vaginal tissue was excised from uninfected, untreated mice in estrus or diestrus, and uninfected mice treated with DMPA or LNG, and processed to assess the gene expression levels of the desmosomal cadherins DSG1 $\alpha$ and DSC1. Mice in diestrus and mice treated with DMPA or LNG showed significantly lower expression DSG1 $\alpha$ and DSC1, but not tight junction protein 1, occludin, or claudin-1 (mean \pm s.d.) (data from two independent experiments with six mice per group). (b) Immunofluorescence staining of vaginal tissue from mice treated in same manner as a assessed levels of DSG1 $\alpha$ protein expression (representative images from two independent experiments with five animals per condition); DSG1 $\alpha$ (green); DAPI (blue) (L denotes vaginal lumen). (c) Quantification of data displayed in $\mathbf{b}$ showed that LNG and DMPA significantly reduced DSG1 $\alpha$ protein expression compared with mice in estrus or diestrus. One-way ANOVA and Tukey's post hoc tests provided statistical comparisons in a and c. DAPI, 4,6-diamidino-2-phenylindole; DMPA, depotmedroxyprogesterone acetate; DSC1, desmocollin; DSG1 $\alpha$, desmoglein-1 $\alpha$; HSV-2, herpes simplex virus type 2; LNG, levonorgestrel; MePRDL, methylprednisolone. A full color version of this figure is available at the Mucosal Immunology journal online.

integrity and barrier function of cutaneous and intestinal epithelium. $^{31,32}$ Compared with vaginal tissue from estrusstage mice, we found that levels of DSG1 $\alpha$ and DSC1 gene expression were significantly downregulated in diestrus-stage and progestin-treated mice (Figure 3a), whereas detection of DSG1 $\alpha$ protein was most significantly reduced by progestin treatment (Figure $\mathbf{3 b}, \mathbf{c}$ ). On the other hand, DMPA and LNG did not affect the levels of other cell-cell adhesion molecules expressed in genital mucosa, including occludin, claudin-1, or tight junction protein 1 (Figure 3a), while vaginal expression of DSG1 $\alpha$ and DSC1 was unaffected by MePRDL treatment (Supplementary Figure S3). Together, these experiments thus revealed that both DMPA and LNG diminish the genital mucosal barrier; specifically diminishing genital tissue expression of the cell-cell adhesion molecules DSG $1 \alpha$ and DSC1.

\section{DMPA facilitated invasion of endogenous vaginal microbiota into genital tissue}

Although multiple clinical studies reported increased genital tissue inflammation in women using DMPA, ${ }^{33,34}$ no explanation has been provided for this observation. Because of the profound increases in genital mucosal permeability seen in DMPA-treated mice, we posited that DMPA promotes tissue inflammation by facilitating infiltration of the endogenous vaginal microbiota. To test this hypothesis, we utilized a LillieTwort tissue Gram stain and a peptide nucleic acid fluorescence in situ hybridization (PNA FISH) assay to detect bacteria in 

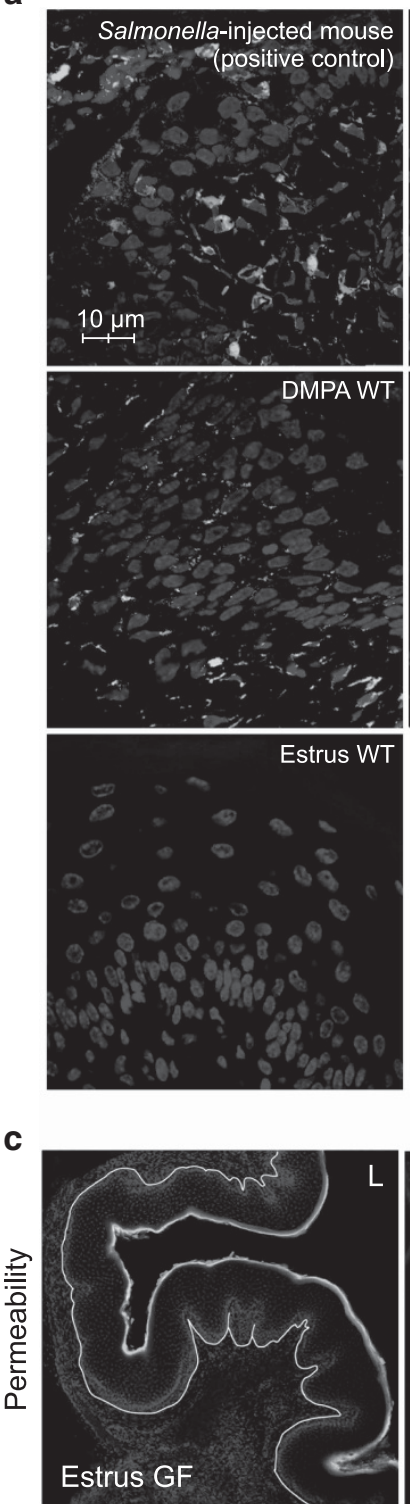

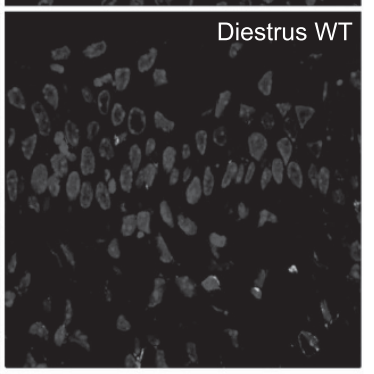

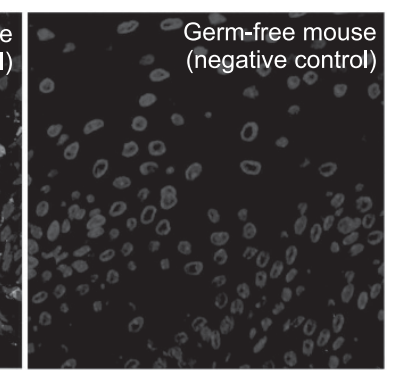
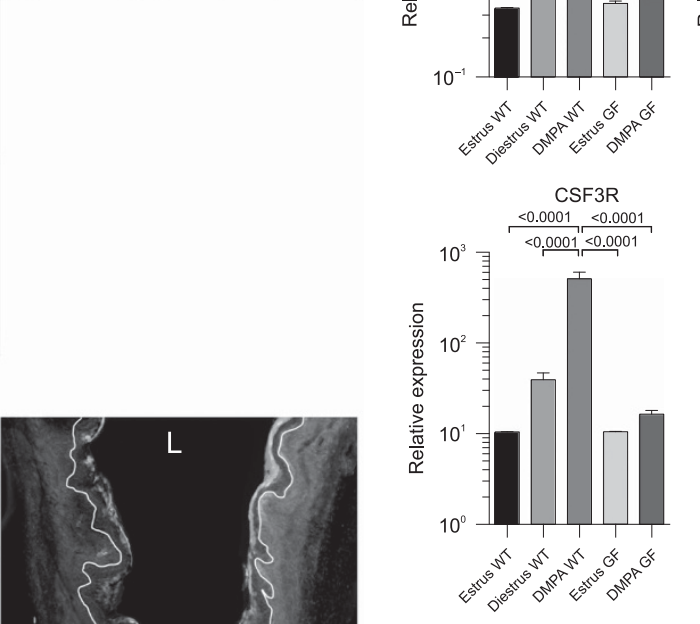

b IL-1 $\beta$

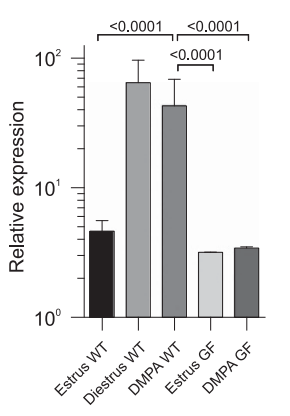

CXCR1
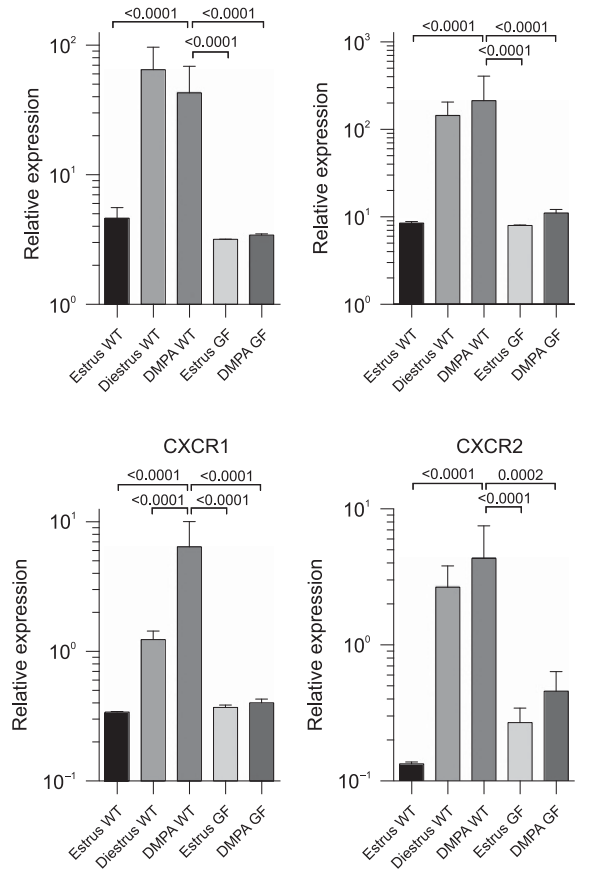

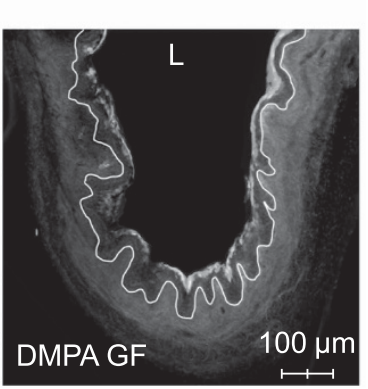

Figure 4 DMPA promoted vaginal tissue inflammation by enhancing tissue invasion of endogenous vaginal microbiota. (a) Representative peptide nucleic acid fluorescence in situ hybridization assay (PNA FISH) images showed a dramatic increase in endogenous bacterial microbiota in the vaginal submucosa of uninfected, DMPA-treated mice (injection of Salmonella into vaginal tissue $1 \mathrm{~h}$ before euthanasia provided positive controls and GF mice provided negative controls); bacteria (green); DAPI (blue) (representative images from two independent experiments with three animals per condition). (b) qRT-PCR analysis of various proinflammatory cytokine and chemokine levels in the vaginal tissue of uninfected WT mice in estrus or diestrus, DMPAtreated mice, GF mice in estrus, or DMPA-treated GF mice showed that genes related to neutrophil infiltration and inflammation were elevated in WT mice in diestrus or after DMPA treatment. However, similar responses were not seen in DMPA-treated GF mice, indicating that inflammatory changes were sequelae to tissue incursion by endogenous microbiota (mean \pm s.d.) (data displayed from two independent experiments with six mice per group). (c) Confocal microscopy images showed that the increases in vaginal mucosal permeability displayed in uninfected, DMPA-treated GF mice were not detected in untreated GF mice in estrus, implying that DMPA-mediated disruption of the mucosal barrier was not sequelae to increased tissue inflammation. Representative images from two independent experiments with three animals per condition are shown (L denotes vaginal lumen). Levels of the proinflammatory cytokines and cytokines in b were compared using one-way ANOVA and Dunnett's post hoc test. DAPI, 4,6-diamidino-2phenylindole; DMPA, depot-medroxyprogesterone acetate; GF, germ-free; qRT-PCR, quantitative real-time PCR; WT, wild type. A full color version of this figure is available at the Mucosal Immunology journal online.

vaginal tissues of uninfected mice in estrus or diestrus or uninfected, DMPA-treated mice. As hypothesized, we saw greater numbers of Gram-negative bacteria in the submucosal tissues of diestrus-stage and DMPA-treated mice, with the latter providing the higher intensity signal (Supplementary Figure S4 and Figure 4a). Moreover, vaginal tissues from these mice displayed significantly greater gene expression levels of the proinflammatory cytokine interleukin-1 $\beta$ (IL-1 $\beta$ ), the neutrophil chemoattractant pro-platelet basic protein (also known as CXCL7), the chemokine receptors CXCR1 and CXCR2, and colony stimulating factor 3 receptor (also known as G-CSF) (Figure 4b). Confirming this inflammatory response 
was sequelae to endogenous microbiota infiltration, no DMPA-mediated increases in genital inflammation were seen when germ-free (GF) mice (i.e., mice without vaginal microbiota) were treated with DMPA (Figure 4b). Conversely, LMW molecules readily penetrated into the vaginal mucosa of uninfected, DMPA-treated GF mice (Figure 4c), indicating that DMPA-mediated increases in mucosal permeability were not a consequence of DMPA-mediated increase in vaginal inflammation. Combined, these studies showed that DMPA facilitated endogenous microbiota invasion into genital tissue, and newly revealed that increases in genital inflammation occurred downstream of DMPA-mediated impairment of the mucosal barrier.

\section{Exogenous estrogen rescued DMPA-treated mice from lethal HSV-2 infection}

As mice in estrus (i.e., the estrous cycle stage with peak endogenous estrogen levels) displayed elevated DSG1 $\alpha$ tissue expression, greater mucosal barrier protection, and imperviousness to ivag HSV-2 infection, we posited mice concomitantly treated with DMPA and a commercially available vaginal cream containing conjugated estrogens would demonstrate reduced HSV-2 susceptibility. And as hypothesized, we detected $100 \%$ survival in concomitantly treated mice after lethal HSV-2 challenge (Figure 5a,b). DMPA-treated mice treated systemically with $17-\beta$ estradiol $\left(\mathrm{E}_{2}\right)$ were similarly resistant to ivag HSV-2 infection (Figure 5a,b), indicating that the increased survival produced by ivag estrogen treatment was not an artifact of the cream blocking virus access to the vaginal mucosa. Vaginal mucosal permeability was also decreased in mice treated with DMPA and estrogen, compared with mice receiving DMPA alone (Figure 5d). Conversely, while both systemic and local estrogen treatments increased DSG1 $\alpha$ expression, only estrogen cream affected DSC1 expression (Figure $\mathbf{5 c - e}$ ), results that imply a larger role for DSG1 $\alpha$ in maintaining genital mucosal integrity. Although progesteronemediated decreases in vaginal epithelial keratinization were postulated as a mechanism by which DMPA increases genital infection susceptibility, ${ }^{16,17}$ in our studies, keratinization of the vaginal epithelial was increased in estrus-stage mice only, whereas both estrus-stage mice and mice treated with DMPA and estrogen were resistant to HSV-2 infection (Supplementary Figure S5). Taken together, our results thus suggested that epithelial barrier function has a more important role than keratinization in protecting mice from ivag HSV-2 infection.

\section{DMPA treatment increased permeability of human ectocervical mucosal tissue}

By designing experiments analogous to those performed in mice, we sought to provide clinical relevance for the increased genital mucosal permeability seen in DMPA-treated mice. For these studies, we obtained ectocervical biopsy tissue from women using no hormonal contraceptive and 1 month after these women elected to initiate use of DMPA. Remarkably, in comparison with biopsy tissue collected from women at enrollment, we saw significantly decreased expression of
DSG1 in ectocervical tissue collected after DMPA was initiated (Figure 6a). In results consonant with the increased genital tissue inflammation detected after uninfected mice were treated with DMPA (Figure 4b), ectocervical tissue from women at the follow-up visit displayed significantly elevated expression of numerous proinflammatory molecules, including IL-1 $\beta$, CD14 (the lipopolysaccharide co-receptor), and CD177 (the human neutrophil-specific PECAM-1 ligand) (Figure 6b). And in results consistent with decreased expression of DSG1 and increased levels of proinflammatory molecules, mucosal permeability was significantly increased in ectocervical tissue collected from women 1 month after DMPA injection (Figure 6c,d). Interestingly, this increased mucosal permeability positively correlated with serum MPA levels that were detected in women at the follow-up visit (Figure 6e,f). Combined, our clinical data newly revealed alterations in genital tissue inflammation, cell-cell adhesion molecule expression, and mucosal permeability in ectocervical tissue of women initiating DMPA use that were exactly analogous to changes found in genital tissue of DMPA-treated mice.

\section{DISCUSSION}

STI and unplanned pregnancy are highly interconnected problems that need solved in tandem, as countries with the largest STI prevalence often have higher infant and maternal mortality rates and greater need for effective contraception. More than 2 decades ago, DMPA was identified as a risk factor for STI acquisition, but this result was not always seen in subsequent studies, and there is persistent concern that data interpretation from clinical studies is confounded by higher frequencies of unprotected sex among couples using hormonal contraception. Although most prior studies focused on the relationship between DMPA use and HIV acquisition, DMPA was also recently identified as a risk factor for male-to-female sexual transmission of HSV $-2 .^{35}$ To define DMPA-mediated effects on anti-viral host defense in the female genital tract, herein we utilized an experimental system in which DMPAtreated mice are uniformly susceptible to genital HSV-2 infection. Using this system, we discovered that treatment greatly decreased genital tissue cell-cell adhesion molecule expression, and dramatically increased genital mucosal permeability to LMW molecules, infectious virus particles, inflammatory cells, and endogenous microbiota.

These DMPA-mediated changes in genital mucosal permeability may have particular relevance for viral pathogenesis, as recent work in non-human primates showed depth of virus penetration into squamous epithelium (e.g., ectocervix) is directly proportional to epithelial permeability. ${ }^{36}$ Although HSV-2 induced 100\% mortality in DMPA-treated mice in our study, mice infected during diestrus were only slightly more susceptible to infection than mice infected during estrus. Of note, differential susceptibility was also observed when nonhuman primates were infected with simian immunodeficiency virus after treatment with DMPA or during the luteal stage of their menstrual cycle. ${ }^{37}$ These experimental findings imply that 
a

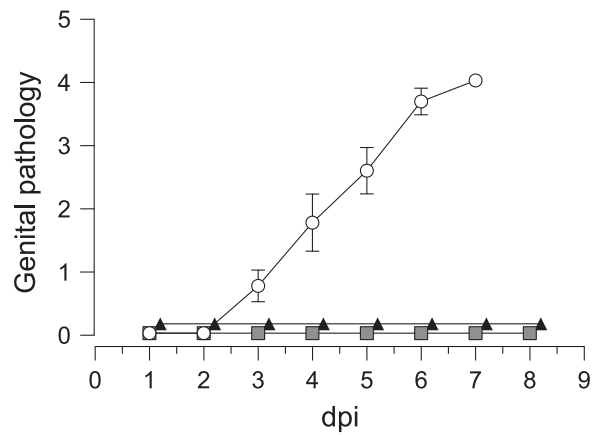

-o- DMPA b

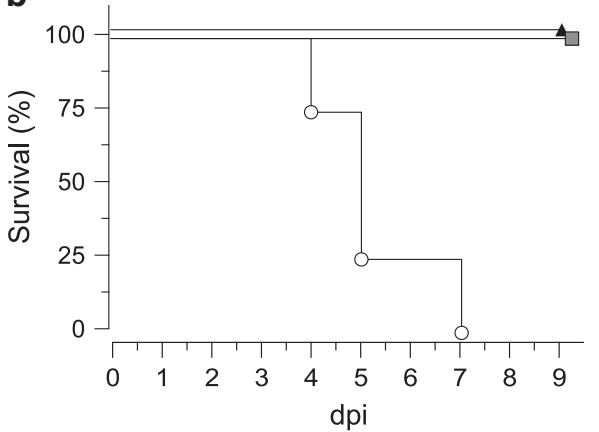

- DMPA + $E_{2}$ sc c
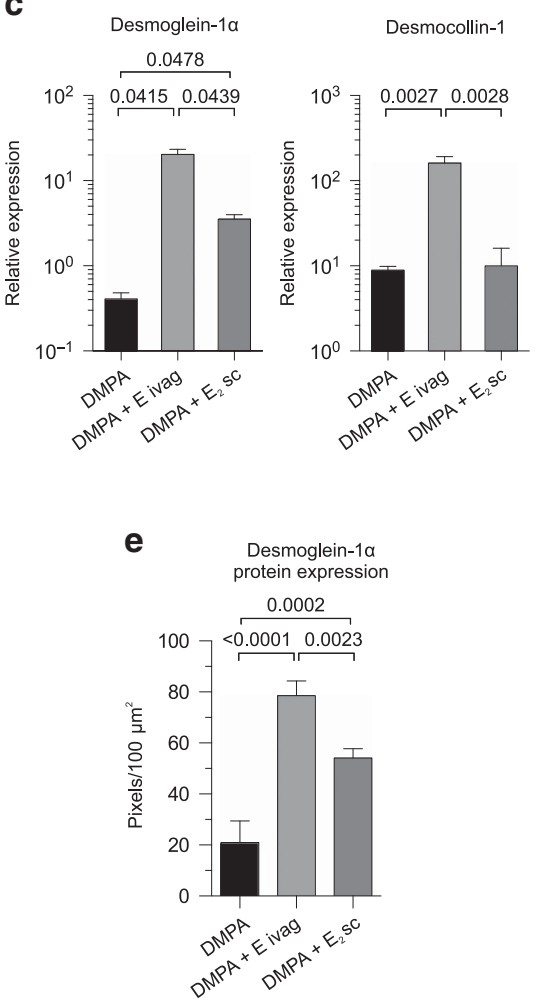

d

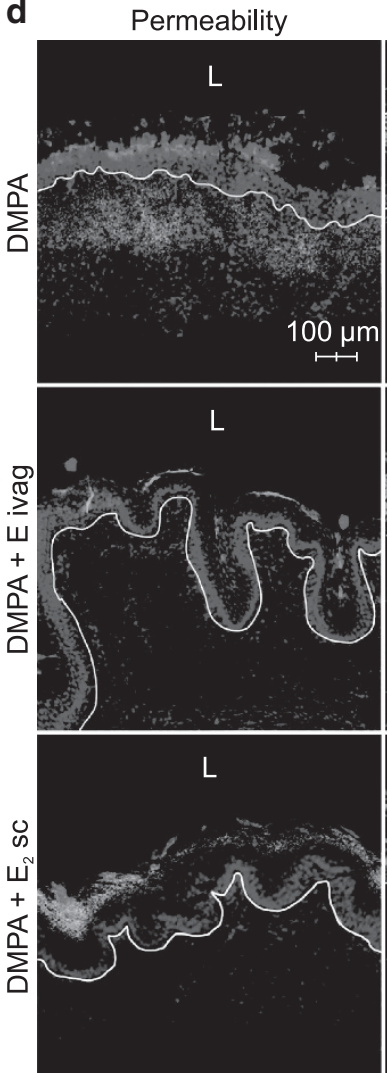

Desmoglein-1 $\alpha$

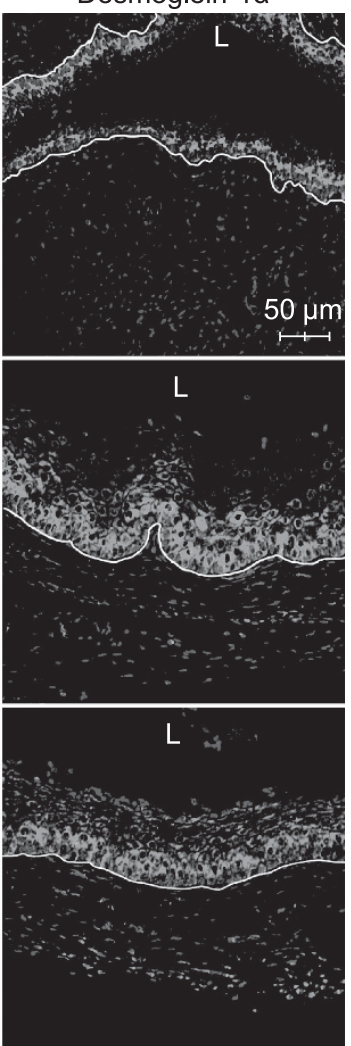

Figure 5 Exogenous estrogen (E) restored DSG1 $\alpha$ expression and reduces susceptibility of DMPA-treated mice to ivag HSV-2 infection. (a, b) DMPA-

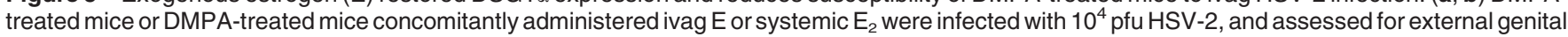
pathology and mortality. Pathology and survival curve data demonstrated both $E$ treatments abrogated development of morbidity and mortality seen in DMPA-treated mice (data shown representative of two independent experiments containing five mice per group). (c) Consistent with the effects of $E$ treatments on mouse survival after ivag HSV-2 infection, DMPA-mediated decreases in vaginal tissue DSG1 $\alpha$ expression were restored by local E and systemic $E_{2}$ treatments (mean \pm s.d.) (data from two independent experiments with six mice per group). (d) Both DSG1 $\alpha$ protein expression and epithelial permeability were re-established by E treatments of DMPA-treated mice (representative figures from two independent experiments and three mice per condition), for left panels: LY (green); DR (red); DAPI (blue), for right panels, DSG1 $\alpha$ (green); DAPI (blue); (L denotes vaginal lumen). (e) DSG1 $\alpha$ protein expression shown in $\mathbf{d}$ was quantified as defined in Methods (mean \pm s.d.). Results from two independent experiments with three mice per group are shown. Kruskal-Wallis test with Dunn's post hoc tests was used for a; one-way ANOVA and Tukey's post hoc tests were used for comparisons in $\mathbf{c}$ and $\mathbf{e}$ and log-rank tests were used to compare cumulative survival incidence after ivag HSV-2 infection in b. DAPI, 4,6-diamidino-2-phenylindole; DMPA, depot-medroxyprogesterone acetate; DR, dextran Texas-Red; DSG1 $\alpha$, desmoglein-1 $\alpha$; HSV-2, herpes simplex virus type 2; ivag, intravaginally; LY, Lucifer yellow $\mathrm{CH}$, lithium salt. A full color version of this figure is available at the Mucosal Immunology journal online.

higher levels of endogenous progesterone enhance susceptibility to viral genital pathogens, but that more prolonged exposure to unopposed exogenous progestin represents the greater risk for virus acquisition. They also spotlight a significant obstacle for clinical studies seeking to discriminate between exogenous progestin-mediated risks for virus acquisition vs. those associated with menstrual cycle stages, and highlight the need for animal models to precisely define 

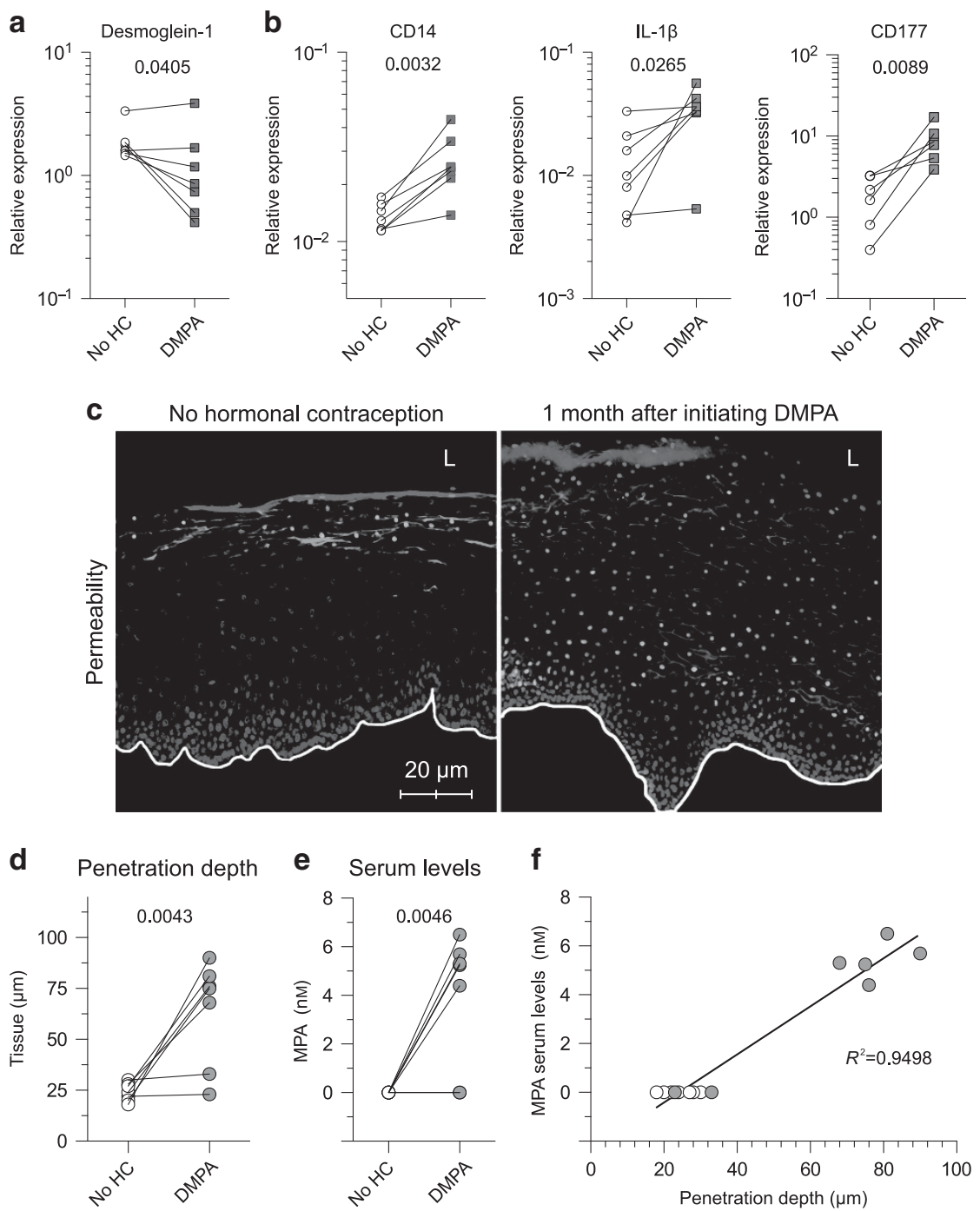

Figure 6 Women initiating DMPA use displayed significant increases in ectocervical mucosal permeability. (a) Ectocervical biopsy tissues collected from women using no form of hormonal contraceptive (enrollment visit, no $\mathrm{HC}$ ) and 1 month after initiating DMPA (follow-up visit) revealed significantly reduced DSG1 expression in tissues obtained after treatment (panel displays relative gene expression levels before and after DMPA initiation for seven women). (b) Analogous to changes seen in uninfected mice after DMPA treatment, DMPA use correlated with increased ectocervical tissue inflammation at the follow-up visit (panel displays relative expression levels of genes related to inflammation and neutrophil migration for seven women before and after initiating DMPA use). (c) Representative images from ectocervical biopsy tissue samples incubated with LY and DR revealed DMPA-mediated loss of mucosal barrier function that was notably similar to that seen in DMPA-treated mice $(n=7)$ (L denotes the vaginal lumen). (d) Quantification of LY penetration depth into ectocervical mucosa of tissue biopsies from women before and after their initiation of DMPA use $(n=7)$. (e) MPA serums levels detected in women at the enrollment and follow-up visits. (f) Linear regression analysis identified a positive correlation between MPA serum concentration and LY penetration depth into ectocervical mucosal tissue ( $R^{2}$ value reported). Paired Student's $t$-tests were used to provide statistical comparisons in a, b, d, and e. DAPI, 4,6-diamidino-2-phenylindole; DMPA, depot-medroxyprogesterone acetate; DR, dextran Texas-Red; DSG1 $\alpha$, desmoglein-1 $\alpha$; HC, hormonal contraceptive; HSV-2, herpes simplex virus type 2; MPA, medroxyprogesterone acetate; LY, Lucifer yellow $\mathrm{CH}$, lithium salt. A full color version of this figure is available at the Mucosal Immunology journal online.

the strength of association between LARC use and STI susceptibility.

Although vaginal mucosa thickness was equivalent among mice in diestrus and after progestin treatment in our investigation, HSV-2-induced mortality was highest among progestin-treated mice. Such results mirror clinical findings in which comparable vaginal mucosal thicknesses were seen between women in their luteal phase and women using DMPA, ${ }^{38-40}$ and provide further support that progestinmediated increases in genital mucosal permeability have an important role in promoting uniform susceptibility of mice to ivag HSV-2 infection. In conjunction with our use of a mouse model of HSV-2 infection, we performed studies with uninfected GF mice that showed DMPA-mediated increases in tissue inflammation were a consequence of DMPA-mediated weakening of barrier function. Because prior clinical studies reported similar increases in genital tissue inflammation after women initiated use of DMPA, ${ }^{33,34}$ we anticipated other findings from our mouse model would recapitulate upon interrogation of the ectocervical biopsy tissues collected from women in our investigation. And as predicted by the mouse data, our clinical findings revealed that DMPA use was 
associated with significantly decreased cell-cell adhesion molecule expression and significant increases in ectocervical mucosal permeability and inflammation. Interestingly, both our animal model and clinical findings are supported by recent studies in which women using DMPA displayed significantly higher frequencies of T cells, macrophages, and HLA-DR ${ }^{+}$and CCR $5^{+}$cells in vaginal mucosal tissue, ${ }^{41}$ and CCR $5^{+}$T cells in the peripheral blood. ${ }^{42}$

Diminished barrier protection in DMPA-treated mice in our investigation was produced with pharmacologically relevant doses of the drug, ${ }^{43,44}$ and we detected DMPA-mediated effects in human ectocervical tissue strikingly similar to the changes seen in mice. However, we do not know whether the progestinmediated increases in virus susceptibility seen in mice are also produced in women. Likewise, our findings do not exclude the possibility that DMPA weakens other mechanisms of innate host defense such as mucus or antimicrobial peptide production or macrophage or natural killer cell function. On the other hand, our mouse data minimize the possibility that greater activation of glucocorticoid signaling pathways increased susceptibility of progestin-treated mice to HSV-2 infection. Specifically, while we detected $100 \%$ mortality and no changes in vaginal tissue expression of glucocorticoid-specific genes in mice treated with the PR agonist LNG, MePRDL treatment significantly modulated glucocorticoid signaling pathways without enhancing susceptibility to genital infection. Therefore, our mouse studies provide novel indication that DMPA- and LNG-containing LARC similarly impair mucosal barrier protection. This possibility is especially provocative as use of LNG-IUD in the United States has doubled in the last 5 years, ${ }^{45}$ and the American Academy of Pediatrics now recommends this LARC as a first choice for adolescent women; ${ }^{46}$ individuals particularly vulnerable for STI acquisition. Although implications for our findings may therefore be substantial, clearly additional basic, translational, and clinical studies are needed to define the effects of DMPA and LNG-IUD on susceptibility to genital tract pathogens.

In addition to newly defining progestin-mediated effects on genital mucosal permeability, our rescue of DMPA-treated mice with local estrogen administration offers indication that combined use of DMPA and an estradiol-releasing vaginal device may provide the basis for contraceptive strategies less likely to enhance susceptibility to genital pathogens. Interestingly, our findings are congruent with earlier reports that showed estrogen promoted epithelial repair, ${ }^{47}$ and that estrogen-treated non-human primates were more resistant to genital simian immunodeficiency virus infection. ${ }^{48,49}$ Although current dogma maintains sexual intercourse may diminish any enhanced barrier protection provided by estrogen-mediated increases in epithelial keratinization, our results imply that estrogen-mediated increases in epithelial integrity and barrier function are the more vital anti-viral host defense. Therefore, the combined use of a progestin-containing injectable contraceptive and an estradiol-containing vaginal delivery device may offer a pragmatic approach for hormonal contraception. In one possible scenario, women would receive
DMPA and an estradiol-releasing vaginal ring (both are commercially available and continuously release hormones for 3 months) that also releases an antiviral microbicide. Although presently only a hypothetical alternative, determining the safety and efficacy of such an approach warrants further investigation.

\section{METHODS}

Evaluation of human ectocervical biopsy tissue before and after initiating DMPA. Procedures were performed in accordance with the Helsinki Declaration and approved by The Ohio State University (OSU) Biomedical Sciences Institutional Review Board (ClinicalTrials.gov Identifier NCT02011711). Healthy, non-pregnant women 18-25 years of age with self-reported history of regular menses, no hormonal contraceptive use in the prior 6 months, and making the personal decision to initiate DMPA use were eligible to participate. After obtaining written consent, enrollment visits were scheduled to concur with participants' late follicular menstrual cycle stage. At the enrollment visit, three ectocervical biopsies were obtained from the 12 o'clock, 4 o'clock, and 8 o'clock positions on the ectocervix. Biopsy tissue was placed in RNAlater (Life Technologies, Carlsbad, CA) or transport media containing RPMI-1640 (Cellgro, Mediatech, Manassas, VA) supplemented with $10 \% \mathrm{AB}$ human serum (Atlanta Biologicals, Flowery Branch, GA), $2 \mathrm{~mm}$ L-glutamine, $1 \mathrm{~mm}$ sodium pyruvate, non-essential amino acids, $100 \mathrm{U} \mathrm{ml}^{-1}$ penicillin, $100 \mu \mathrm{g} \mathrm{ml}^{-1}$ streptomycin, $50 \mu \mathrm{g} \mathrm{ml}^{-1}$ gentamycin, and $50 \mu \mathrm{M} 2-\mathrm{ME}$ (all from Cellgro). Women were scheduled to receive a $150-\mathrm{mg}$ DMPA injection at the beginning of next menstrual cycle, and asked to return to clinic 30-45 days after treatment. At this visit, three biopsies were collected from the 2 o'clock, 6 o'clock, and 10 o'clock positions of the ectocervix and processed identically as at enrollment. During follow-up visits, earlier biopsy sites were assessed, and without exception, these sites appeared fully healed. At each visit, peripheral blood was collected to obtain serum, which was used to measure serum MPA concentrations as previously described, ${ }^{43}$ and genital specimens were collected that confirmed the absence of Neisseria gonorrhoeae, Chlamydia trachomatis, and Trichomonas vaginalis infections by nucleic acid amplification testing and the presence of normal vaginal flora by Gram stain evaluation.

Treatment of mice with exogenous sex steroids. The OSU Institutional Animal Care and Use Committee approved all mouse experiments. As indicated, Pg (Calbiochem, La Jolla, CA), LNG, mifepristone, or estradiol $\left(\mathrm{E}_{2}\right)$ (all from Sigma-Aldrich, St Louis, MO) underwent glass-to-glass homogenization for suspension in phosphate-buffered saline (PBS) $15 \mathrm{~min}$ before in vivo administration. Likewise, sustained-release injectable DMPA (Depo-Provera) and MePRDL (Depo-Medrol) (both from Pharmacia and Upjohn, New York, NY) were diluted in PBS before injection. Conjugated estrogen vaginal cream (Premarin) (Wyeth Pharmaceuticals, Philadelphia, PA) was ivag administered. As indicated, 6- to 8-week-old female wild-type (WT) C57BL/6J mice (The Jackson Laboratory, Bar Harbor, ME) were subcutaneously (s.c.) injected with $1 \mathrm{mg}$ DMPA or $2 \mathrm{mg}$ MePRDL (i.e., a dose shown to be immunosuppressive in mice ${ }^{50}$ ) 5 days before infection or evaluated on the day of infection for estrous cycle stage, as previously described. ${ }^{51}$ Also as indicated, mice were s.c. injected with $1 \mathrm{mg}$ Pg daily for 5 days before and 3 days after infection. For LNG administration, mice were treated daily from 5 days before until 3 days after infection with $2.5 \mu \mathrm{g}$ of LNG suspended in $10 \mu \mathrm{l}$ of PBS (for ivag administration) or $1 \mathrm{mg}$ LNG suspended in $100 \mu \mathrm{l}$ of PBS (for s.c. injection). Where indicated, DMPA-treated mice were s.c. administered $1 \mathrm{mg}$ RU486 or $1 \mathrm{mg} \mathrm{E} \mathrm{E}_{2}$ for 5 days before and 3 days after infection, or ivag treated with conjugated estrogen vaginal cream daily for 5 days before infection. Also as indicated, C57BL/6 female GF mice were assessed for estrous cycle stage, or injected s.c. with $1 \mathrm{mg}$ DMPA 5 days before euthanasia. 
HSV-1 and HSV-2 infections. Mice were sedated with $0.18 \mathrm{mg}$ xylazine (Lloyd Laboratories, Shenandoah, IA) and $1.8 \mathrm{mg}$ ketamine hydrochloride (JHP Pharmaceuticals, LLC Rochester, MI), and ivag infected with $10^{4}$ plaque-forming units (pfu) of WT HSV-2 333 (provided by Dr Robert Hendricks, University of Pittsburgh) in $10 \mu \mathrm{l}$ of RPMI. Genital pathology was evaluated daily using a previously described 5 -point scale: 0 , no pathology; 1 , mild vulvar erythema; 2 , moderate vulvar erythema; 3, severe vulvar erythema and perineal fur loss; 4 , perineal ulceration; 5 , extension of perineal ulceration and fur loss to surrounding tissue, ${ }^{52}$ with mice killed if scores $\geq 4$ or encephalopathic changes were identified. Other mice were similarly sedated and ivag infected with $3 \times 10^{6}$ pfu of HSV-1q-GPF ${ }^{23}$ in $10 \mu \mathrm{l}$ of RPMI, and killed 1, 12, or $24 \mathrm{~h}$ later. This infectious inoculum was chosen to enable visualization of replicating virus by confocal microscopy. Genital tissues were harvested, fixed with $4 \%$ methanolfree formaldehyde (Thermo Scientific, Rockford, IL), and embedded in $6 \%$ low melting point agarose (Invitrogen, Carlsbad, CA). Next, $250-300 \mu \mathrm{m}$ sections were obtained using a vibratome, and permeabilized with $0.2 \%$ Triton X-100 (Sigma-Aldrich) solution in PBS, stained with 4,6-diamidino-2-phenylindole (DAPI) (Sigma-Aldrich), embedded in mounting media (Vector Laboratories, Burlingame, CA), and evaluated for viral spread with a FV1000 spectral confocal system (Olympus, Center Valley, PA). Confocal microscopy images were acquired by sequential scanning to prevent fluorescence crossover.

Immunofluorescence staining. As indicated, excised vaginas were placed in formaldehyde and paraffin embedded and $10 \mu \mathrm{m}$ tissue sections were mounted on glass slides and de-paraffinized by sequential immersion in $100 \%$ xylene, $100 \%$ ethanol, $96 \%$ ethanol, and sterile DEPC-treated water. Antigen retrieval was performed using $10 \mathrm{~mm}$ sodium citrate buffer ( $\mathrm{pH}$ 6.0) containing $0.05 \%$ Tween-20 (both from Sigma-Aldrich) $\left(20 \mathrm{~min}\right.$ at $\left.95^{\circ} \mathrm{C}\right)$. After three PBS washes, sections were incubated overnight with $10 \%$ normal donkey serum (Abcam, Cambridge, MA) at $4{ }^{\circ} \mathrm{C}$, incubated $1 \mathrm{~h}$ at ambient temperate with rabbit anti-desmoglein-1 (clone EPR6766(B)), washed, and incubated $1 \mathrm{~h}$ with AlexaFluor 488-labeled donkey anti-rabbit IgG labeled (both antibodies from Abcam) (all antibodies were diluted in PBS with $1 \%$ BSA and $0.05 \%$ Tween-20). Sections were stained with DAPI, and evaluated by confocal microscopy for relative expression of DSG1 $\alpha$ protein (defined by calculating pixel numbers per $100 \mu \mathrm{m}^{2}$ ) using the ImageJ software. ${ }^{53}$ To evaluate relative DSG1 $\alpha$ protein expression, images were acquired using identical parameters and their contrast altered equally (investigators performing such measurements were blinded to study group designation).

Genital tract permeability assays. Mice were sedated, and administered $10 \mu \mathrm{l}$ of a PBS solution containing $62.5 \mu \mathrm{g}$ of dextran TexasRed (DR) $(\mathrm{MW}=70,000 \mathrm{Da})$ and $50 \mu \mathrm{g}$ of Lucifer yellow $\mathrm{CH}$, lithium salt $(\mathrm{LY})(\mathrm{MW}=457.2 \mathrm{Da})$ (both from Life Technologies). After $45 \mathrm{~min}$, mice were killed, vaginas excised, and tissues fixed in formaldehyde, agarose-embedded, DAPI stained, and assessed by confocal microscopy. Confocal microscopy images were acquired by sequential scanning to prevent fluorescence crossover. In other studies, untreated syngeneic mice were killed, and spleens harvested and processed into single-cell suspensions, as described elsewhere. ${ }^{43}$ Splenocytes were labeled with $5 \mu \mathrm{M}$ of carboxyfluorescein succinimidyl ester (CellTrace CFSE from Life Technologies) and re-suspended in PBS at $2 \times 10^{6}$ cells per $10 \mu \mathrm{l}$ of PBS. These fluorescently labeled splenocytes were ivag administered to sedated mice (previously treated as indicated). After $12 \mathrm{~h}$, mice were killed and vaginas processed as above. Confocal image analysis was performed to determine the depth of leukocyte infiltration into vaginal tissue.

After collection, fresh human cervical biopsies were placed in transport media, transferred to sterile 96-well plates, and incubated $45 \mathrm{~min}$ in $20 \mu \mathrm{l}$ of PBS containing LY and DR at the concentrations utilized in the mouse studies. Likewise, biopsies were fixed and embedded in agarose as described for murine tissue. For microscopic evaluation, stratified squamous epithelium, lamina propria, and connective tissue were identified in each ectocervical sample under $\times 100$ magnification. Images were obtained at $\times 400$ magnification and used to quantify penetration of LY into ectocervical tissue.

Light microscopy. Fixed, paraffin-embedded mouse vaginal tissue was sectioned and de-paraffinized for hematoxylin and eosin (H\&E) or Lillie-Twort (Gram) staining. Light microscopy was used to calculate epithelial thickness (excluding cornified layers) by evaluating five random fields (with five measurements per field) using the CellSens Software (Olympus) (investigators performing these measurements were blinded to study group designations).

Peptide nucleic acid fluorescence in situ hybridization assay. As described above, paraffin-embedded vaginal tissues from WT or GF mice were sectioned and de-paraffinized. For positive controls, WT mice were sedated for Salmonella enterica subsp. enterica (ATCC 14028 ) injection into vaginal tissue, and killed $1 \mathrm{~h}$ later to harvest vaginal tissue. To provide negative controls, vaginal tissues were harvested from GF mice. BacUni PNA (universal rRNA bacteria sequence probe) kits (AdvanDx, Woburn, MA) were used to identify bacteria using the manufacturer's instructions (standardized controls provided in kits were run for quality assurance), sections were counterstained with DAPI, and evaluated by confocal microscopy (investigators performing evaluations were blinded to study group designations).

RNA isolation. Human ectocervical biopsy tissues and murine vaginal tissues were immediately immersed in $1 \mathrm{ml}$ of RNAlater, stored for $24 \mathrm{~h}$ at $4{ }^{\circ} \mathrm{C}$, and then transferred to $-80^{\circ} \mathrm{C}$ freezer. RNA was isolated using the RNeasy Lipid Tissue Kit (Qiagen, Hilden, Germany) following the manufacturer's instructions, and re-suspended in nucleasefree water. RNA was quantified using a NanoDrop spectrophotometer (Thermo Scientific) (all samples displayed 260/280 ratios $>1.80$ ).

Quantitative real time PCR. In mouse vaginal tissue, desmocollin 1 (Mm00496525_m1), desmoglein-1 $\alpha$ (Mm00809994_s1), tight junction protein 1 (Mm00493699_m1), occludin (Mm00500912_m1), claudin-1 (Mm00516701_m1), CXCR1 (Mm00731329_s1), CXCR2 (Mm99999117_s1), colony stimulating factor 3 receptor (Mm00432735_m1), pro-platelet basic protein (CXCL7) (Mm01347901_g1), IL-1 $\beta$ (Mm00434228_m1), glucocorticoid regulated kinase 1 (Mm00441380_m1), glucocorticoid-Induced leucine zipper (Mm00726417_s1), dual specificity protein phosphatase 1 (Mm00457274_g1), GR (Mm00433832_m1) relative gene expression was evaluated using pyruvate carboxylase (Mm00500992_m1) as a housekeeping reference gene. In human ectocervical tissue, IL-1 $\beta$ (Hs01555410_m1), desmoglein-1 (Hs00355084_m1), CD14 (Hs02621496_s1), and CD177 (Hs00360669_m1) relative gene expression was evaluated using glucose-6-phosphate isomerase (Hs00976711_m1) as a housekeeping reference gene ${ }^{54}$ (all primers, Life Technologies). To determine relative gene expression, $50 \mathrm{ng}$ of RNA was mixed with TaqMan RNA-to-Ct 1-Step Kit and adequate primer in a final volume of $10 \mu \mathrm{l}$ per well following the manufacturer's instructions (Life Technologies). Quantitative real-time PCR was performed using a CFX96 Thermal Cycler, and data analyzed using the CFX Manager Software (Bio-Rad Laboratories, Hercules, CA).

Statistical considerations. Statistical analyses were performed using the Prism 6 software (GraphPad, La Jolla, CA). For each experiment, normal distribution was tested by D'Agostino \& Pearson omnibus test or evaluation of the residuals (when experimental sample numbers were $<8$ ). When comparing two groups, differences for paired samples were compared using the paired Student's $t$-test, and unpaired samples were compared using the unpaired Student's $t$-tests. For multiple comparisons, one-way ANOVA with Dunnett's or Tukey's post hoc test was used for parametric distributions, and Kruskal-Wallis test with Dunn's post hoc test was used for non-parametric distribution (i.e., depending on data distribution). To compare scores for the 
development of genital pathology, the area under the curve was calculated for each mouse and then multiple group comparisons performed, as indicated above. Kaplan-Meier survival curves and logrank test were used to compare cumulative survival incidence after ivag HSV-2 infection. Linear regression analysis was performed between MPA serum concentrations and depth of LY tissue penetration into human ectocervical tissue $\left(R^{2}\right.$ value reported and residuals evaluated $^{55}$ ). For all presented comparisons, $P$ values $\leq 0.05$ were considered statistically significant.

SUPPLEMENTARY MATERIAL is linked to the online version of the paper at http://www.nature.com/mi

\section{ACKNOWLEDGMENTS}

We thank study participants and the clinical and laboratory staff who contributed to this work, particularly Kathryn Martin, Cindy Overholts, Amanda Luff, Haley Steiner, and Jocelyn Gilmore. We acknowledge Juan Gonzalez, Brian Ahmer, Mark Landon, and Ann Thompson for thoughtful discussion, Narender Kumar for providing expertise with serum MPA measurements, and technical support provided by The OSU Comparative Pathology and Mouse Phenotyping Shared Resource (NIH grant P30 CA016058), University Laboratory Animal Resources, and Campus Microscopy and Imaging Facility. Study funding was provided by the NICHD (grant R01HD072663) and the OSU College of Medicine.

\section{AUTHOR CONTRIBUTIONS}

Experimental design, data acquisition, data analysis, and data interpretation were performed by N.E.Q.C., R.D.V.M., and T.L.C.; critical laboratory reagents were provided by P.N.B., L.H-S., and B.K.; W.T. supervised clinical research; S.D.P. performed a portion of the experiments and data analysis. Manuscript was initially drafted by N.E.Q.C. and R.D.V.M., all authors significantly contributing to its final form.

\section{DISCLOSURE}

The authors declared no conflict of interest.

(c) 2016 Society for Mucosal Immunology

\section{REFERENCES}

1. Quinn, T.C. \& Overbaugh, J. HIV/AIDS in women: an expanding epidemic. Science 308, 1582-1583 (2010)

2. Morrison, C.S. et al. Hormonal contraceptive use, cervical ectopy, and the acquisition of cervical infections. Sex Transm. Dis. 31, 561-567 (2004).

3. Baeten, J.M. et al. Hormonal contraceptive use, herpes simplex virus infection, and risk of HIV-1 acquisition among Kenyan women. AIDS 21, 1771-1777 (2007)

4. Morrison, C.S. et al. Hormonal contraception and HIV acquisition: reanalysis using marginal structural modeling. AIDS 24, 1778-1781 (2010).

5. Heffron, R. et al. Partners in Prevention HSV/HIV Transmission Study Team. Use of hormonal contraceptives and risk of HIV-1 transmission: a prospective cohort study. Lancet Infect. Dis. 12, 19-26 (2012).

6. Warner, P. Concerns regarding design, analysis, and interpretation of the Morrison study on hormonal contraceptive use and acquisition of cervical infections. Sex Transm. Dis. 32, 644. author reply 645 (2005).

7. Gray, R.H. Use of hormonal contraceptives and risk of HIV-1 transmission. Lancet Infect. Dis. 12, 507 (2012).

8. Hubacher, D. Use of hormonal contraceptives and risk of HIV-1 transmission. Lancet Infect. Dis. 12, 508 (2012).

9. Morrison, C.S. \& Nanda, K. Hormonal contraception and HIV: an unanswered question. Lancet Infect. Dis. 12, 2-3 (2012).

10. Ralph, L.J., McCoy, S.I., Hallett, T. \& Padian, N. Next steps for research on hormonal contraception and HIV. Lancet 382, 1467-1469 (2013).

11. Feldblum, P.J. et al. Randomized assignment to copper IUD or depotmedroxyprogesterone acetate: feasibility of enrollment, continuation and disease ascertainment. Contraception 72, 187-191 (2005).

12. Stringer, E.M. et al. A randomized trial of the intrauterine contraceptive device vs hormonal contraception in women who are infected with the human immunodeficiency virus. Am. J. Obstet. Gynecol. 197, 144.e1-e8 (2007).

13. Shelton, J.D. Use of hormonal contraceptives and risk of HIV-1 transmission. Lancet Infect. Dis. 12, 507-508 (2012).

14. McCoy, S.I., Ralph, L.J., Padian, N.S. \& Minnis, A.M. Are hormonal contraceptive users more likely to misreport unprotected sex? Evidence from a biomarker validation study in Zimbabwe. AIDS Behav. 18, 2259 2264 (2014).

15. Murphy, K., Irvin, S.C. \& Herold, B.C. Research gaps in defining the biological link between HIV risk and hormonal contraception. Am. J. Reprod. Immunol. 72, 228-235 (2014).

16. McNicholl, J.M., Henning, T.C., Vishwanathan, S.A. \& Kersh, E.N. Non-human primate models of hormonal contraception and HIV. Am. J. Reprod. Immunol. 71, 513-522 (2014).

17. Marx, P.A. et al. Progesterone implants enhance SIV vaginal transmission and early virus load. Nat. Med. 2, 1084-1089 (1996).

18. Khanna, K.V. et al. Vaginal transmission of cell-associated HIV-1 in the mouse is blocked by a topical, membrane-modifying agent. J. Clin. Invest. 109, 205-211 (2002).

19. Morrison, S.G. \& Morrison, R.P. A predominant role for antibody in acquired immunity to chlamydial genital tract reinfection. J. Immunol. 175, 75367542 (2005).

20. Roberts, J.N. et al. Genital transmission of HPV in a mouse model is potentiated by nonoxynol-9 and inhibited by carrageenan. Nat. Med. 13 857-861 (2007)

21. Whaley, K.J., Barratt, R.A., Zeitlin, L., Hoen, T.E. \& Cone, R.A. Nonoxynol-9 protects mice against vaginal transmission of genital herpes infections. J. Infect. Dis. 168, 1009-1011 (1993).

22. Kaushic, C., Ashkar, A.A., Reid, L.A. \& Rosenthal, K.L. Progesterone increases susceptibility and decreases immune responses to genital herpes infection. J. Virol. 77, 4558-4565 (2003).

23. Yoo, J.Y. et al. Antitumor efficacy of 34.5ENVE: a transcriptionally retargeted and "Vstat120"-expressing oncolytic virus. Mol. Ther. 20, 287-297 (2012).

24. Benagiano, G., Gabelnick, H. \& Farris, M. Contraceptive devices: intravaginal and intrauterine delivery systems. Expert Rev. Med. Devices 5, 639-654 (2008).

25. Africander, D., Verhoog, N. \& Hapgood, J.P. Molecular mechanisms of steroid receptor mediated actions by synthetic progestins used in HRT and contraception. Steroids 76, 636-652 (2011).

26. Stanczyk, F.Z., Hapgood, J.P., Winer, S. \& Mishell, D.R. Jr. Progestogens used in postmenopausal hormone therapy: differences in their pharmacological properties, intracellular actions, and clinical effects. Endo. Rev. 34, 171-208 (2013).

27. Kadmiel, M. \& Cidlowski, J.A. Glucocorticoid receptor signaling in health and disease. Trends Pharmacol. Sci. 34, 518-530 (2013).

28. Whaley, K.J. \& Mayer, K.H. Strategies for preventing mucosal cellassociated HIV transmission. J. Infect. Dis. 15, S674-S680 (2014).

29. Dinh, M.H., Okocha, E.A., Koons, A., Veazey, R.S. \& Hope, T.J. Expression of structural proteins in human female and male genital epithelia and implications for sexually transmitted infections. Biol. Reprod. 86, 1-6 (2012).

30. Blaskewicz, C.D., Pudney, J. \& Anderson, D.J. Structure and function of intercellular junctions in human cervical and vaginal mucosal epithelia. Biol. Reprod. 85, 97-104 (2001).

31. Green, K.J. \& Simpson, C.L. Desmosomes: new perspectives on a classic. J. Invest. Dermatol. 127, 2499-2515 (2007).

32. Sherrill, J.D. et al. Desmoglein-1 regulates esophageal epithelial barrier function and immune responses in eosinophilic esophagitis. Mucosal Immunol. 7, 718-729 (2014).

33. Goldfien, G.A. et al. Progestin-containing contraceptives alter expression of host defense-related genes of the endometrium and cervix. Reprod. Sci. 22, 814-828 (2015).

34. Deese, J. et al. Injectable progestin-only contraception is associated with increased levels of pro-inflammatory cytokines in the female genital tract. Am. J. Reprod. Immunol. 74, 357-367 (2015).

35. Grabowski, M.K. et al. Use of injectable hormonal contraception and women's risk of herpes simplex virus type 2 acquisition: a prospective study of couples in Rakai, Uganda. Lancet Glob. Health 3, e478-e486 (2015).

36. Carias, A.M. et al. Defining the interaction of HIV-1 with the mucosal barriers of the female reproductive tract. J. Virol. 87, 11388-11400 (2013). 
37. Sodora, D.L., Gettie, A., Miller, C.J. \& Marx, P.A. Vaginal transmission of SIV: assessing infectivity and hormonal influences in macaques inoculated with cell-free and cell-associated viral stocks. AIDS Res. Hum. Retroviruses 14, S119-S123 (1998).

38. Mauck, C.K. etal. The effect of one injection of Depo-Provera on the human vaginal epithelium and cervical ectopy. Contraception 60, 15-24 (1999).

39. Bahamondes, L. et al. The effect upon the human vaginal histology of the long-term use of the injectable contraceptive Depo-Provera. Contraception 62, 23-27 (2000).

40. Mitchell, C.M. et al. Long-term effect of depot medroxyprogesterone acetate on vaginal microbiota, epithelial thickness and HIV target cells. J. Infect. Dis. 210, 651-655 (2014).

41. Chandra, N. et al. Depot medroxyprogesterone acetate increases immune cell numbers and activation markers in human vaginal mucosal tissues. AIDS Res. Hum. Retroviruses 29, 592-601 (2013).

42. Sciaranghella, G. et al. CCR5 expression levels in HIV-uninfected women receiving hormonal contraception. J. Inf. Dis. 212, 1397-1401 (2015).

43. Vicetti Miguel, R.D. et al. Dendritic cell activation and memory cell development are impaired among mice administered medroxyprogesterone acetate prior to mucosal herpes simplex virus type 1 infection. J. Immunol. 189, 3449-3461 (2012).

44. Quispe Calla, N.E., Ghonime, M.G., Cherpes, T.L. \& Vicetti Miguel, R.D. Medroxyprogesterone acetate impairs human dendritic cell activation and function. Hum. Reprod. 30, 1169-1177 (2015).

45. Daniels, K., Daugherty, J. \& Jones, J. Current contraceptive status among women aged 15-44: United States, 2011-2013. NCHS Data Brief. 173, 2014.
46. Committee on Adolescence. Contraception for adolescents. Pediatrics 134, e1244-e1256 (2014).

47. Smith, P.J. et al. Effects of estrogen replacement on infarct size, cardiac remodeling, and the endothelin system after myocardial infarction in ovariectomized rats. Circulation 102, 2983-2989 (2000).

48. Smith, S.M. et al. Topical estrogen protects against SIV vaginal transmission without evidence of systemic effect. AIDS 20, 1637-1643 (2009).

49. Smith, S.M., Baskin, G.B. \& Marx, P.A. Estrogen protects against vaginal transmission of simian immunodeficiency virus. J. Infect. Dis. 182, 708-715 (2000).

50. Zhang, M., Xin, H., Duan, Y. \& Atherton, S.S. Ocular reactivation of MCMV after immunosuppression of latently infected BALB/c mice. Invest. Ophthalmol. Vis. Sci. 46, 252-258 (2005).

51. McLean, A.C., Valenzuela, N., Fai, S. \& Bennett, S.A. Performing vaginal lavage, crystal violet staining, and vaginal cytological evaluation for mouse estrous cycle staging identification. J. Vis. Exp. 15, e4389 (2012).

52. Cherpes, T.L. et al. Use of transcriptional profiling to delineate the initial response of mice to intravaginal herpes simplex virus type 2 infection. Viral Immunol. 26, 172-179 (2013).

53. Schneider, C.A., Rasband, W.S. \& Eliceiri, K.W. NIH Image to ImageJ: 25 years of image analysis. Nat. Methods 9, 671-675 (2012).

54. Eisenberg, E. \& Levanon, E.Y. Human housekeeping genes, revisited. Trends Genet. 29, 569-574 (2013).

55. Marill, K.A. Advanced statistics: linear regression, part I: simple linear regression. Acad. Emerg. Med. 11, 87-93 (2004). 